\title{
Applications of Systems Thinking for Scooter Sharing Transportation System
}

\author{
Christina Caches ${ }^{(凶)}$ and Mo Mansouri \\ School of Systems and Enterprises, Stevens Institute of Technology, Castle Point on Hudson, \\ Hoboken, NJ 07030, USA \\ christinamcaches@gmail.com
}

\begin{abstract}
The scooter sharing system is a service that enables individuals to rent electric scooters for short term rides. The scooters are mostly electric motor scooters but there are some that are electric kick start. The purpose of this system is to transport people short distances in a safe and efficient way. This research was done to define and analyze the scooter sharing transportation system. System thinking is applied to understand, engineer and innovate the scooter sharing system. The scooter transportation system is defined as an interdependent constituent system that applies systemic tools and diagrams like systemigrams. These tools allow for the system properties to be studied along with the interrelationships. There will be different models of the scooter transportation system examined. The stakeholders will be examined to view the interconnections within the system.
\end{abstract}

